\title{
Disperse Dyes Based on Thiazole, Their Dyeing Application on Polyester Fiber and Their Antimicrobial Activity
}

\author{
S. K. Zadafiya, J. H. Tailor, and G. M. Malik \\ Department of Chemistry, Navyug Science College, Surat 395009, India \\ Correspondence should be addressed to G. M. Malik; gmmalik2010@gmail.com
}

Received 26 June 2012; Revised 16 October 2012; Accepted 30 October 2012

Academic Editor: Mohamed Afzal Pasha

Copyright (C) 2013 S. K. Zadafiya et al. This is an open access article distributed under the Creative Commons Attribution License, which permits unrestricted use, distribution, and reproduction in any medium, provided the original work is properly cited.

\begin{abstract}
Various diazotized aryl amines were coupled with N-(4-nitrophenyl)-2-[(4-phenyl-1,3-thiazol-2-yl)amino]acetamide to give the corresponding various azo disperse dyes $\left(D_{1}-D_{13}\right)$. These dyes were applied to polyester fiber by HTHP method and their fastness properties were evaluated. Dyes were characterized by IR, elemental analysis, and NMR spectral studies. These dyes showed very good antibacterial and antifungal activities.
\end{abstract}

\section{Introduction}

Disperse dyes are organic colorants with less water solubility and are applied in colloidal dispersions to hydrophobic textile fibers to produce desired color. With increasing use of polyester fibers and their blends, there has been significant increase in the development of disperse dyes because over $90 \%$ of disperse dyes' usage is for the dyeing of polyester and its blends and with significant increase in the world production of polyester fibers compared to other fibers. Many diazo components have been used in the production of disperse dyes in recent time [1-6]. Derivatives of 2-aminothiazole [7-9] have been used as heterocyclic components since long for different disperse dyes. It was our main objective to synthesize [10-13] the disperse dyes consisting thiazole with azo substituent which further utilized to dye some hydrophobic fibers, to characterize, to evaluate their fastness properties. The dyes were screened for their antimicrobial properties because a large number of natural products and drugs comprises of this heterocyclic moiety [14-16].

\section{Experimental}

2.1. Materials and Methods. Melting points were determined in open capillary tubes and are uncorrected. The purity of dyes was determined by thin-layer chromatography (TLC) using silica gel-G-coated Al-plates. The visible absorption spectra were measured using Shimadzu UV-160 PC Spectrophotometer. Infrared spectra were recorded on a Shimadzu FT-IR 8400 S model using KBr pellets. ${ }^{1} \mathrm{H}$ NMR spectra were recorded on a Varian $400 \mathrm{MHz}$ Spectrophotometer using DMSO solvent and TMS as internal reference (chemical shifts in $\delta, \mathrm{ppm})$. Elemental analysis was carried out on Perkin Elmer (USA) 2400 Series instrument. The fastness to light, wash, and sublimation was assessed in accordance with ISO 105. A convenient laboratory method was used for dyeing polyester to employ high temperature $\left(130^{\circ} \mathrm{C}\right)$ and high pressure (24-30 psi.). The dye bath exhaustion (\%E) of the dyed fiber was determined according to the method. The synthesized dyes were screened for their antimicrobial activity using the Kirby-Bauer method. All the compounds were screened for their in vitro antimicrobial activity against bacterial strains such as Escherichia coli, pseudomonas aeruginosa, Staphylococcus aureus, and fungi Candida albicans at $40 \mu \mathrm{g} / \mathrm{mL}$ concentration.

2.2. Preparation of $\mathrm{N}$-(4-Nitrophenyl)-2-[(4-Phenyl-1, 3-Thiazol-2-yl)Amino]Acetamide (2). In $250 \mathrm{~mL}$ R.B.F., 4-nitroaniline $(2.76 \mathrm{gm}, 0.02 \mathrm{~mole})$ in dry benzene $(60 \mathrm{~mL})$ was cooled to $0-5^{\circ} \mathrm{C}$ and $2-3$ drops of TEA were added. Chloroacetyl chloride $(2.26 \mathrm{~mL}, 0.02$ mole) in dry benzene $(20 \mathrm{~mL})$ was slowly added to RBF with vigorous stirring then the reaction mixture was refluxed for 3 hours. Excess of solvent was removed in vacuum and the residue stirred with water 
TABLE 1: Yield, $\lambda$ max, melting points, and nitrogen analysis of disperse dyes.

\begin{tabular}{|c|c|c|c|c|c|c|}
\hline \multirow{2}{*}{ Dye number } & \multirow{2}{*}{$\mathrm{R}$} & \multirow{2}{*}{ Yield (\%) } & \multirow{2}{*}{$\lambda \max (\mathrm{nm})$} & \multirow{2}{*}{ M.P. ${ }^{\circ} \mathrm{C}$} & \multicolumn{2}{|c|}{ Nitrogen (\%) } \\
\hline & & & & & Found & Calcd. \\
\hline $\mathrm{D}_{1}$ & $\mathrm{H}$ & 81 & 456 & 148 & 18.35 & 18.33 \\
\hline $\mathrm{D}_{2}$ & $4-\mathrm{NO}_{2}$ & 72 & 427 & 153 & 19.55 & 19.47 \\
\hline $\mathrm{D}_{3}$ & $3-\mathrm{NO}_{2}$ & 78 & 472 & 142 & 19.51 & 19.47 \\
\hline $\mathrm{D}_{4}$ & $2-\mathrm{CN}, 4-\mathrm{NO}_{2}$ & 58 & 412 & 157 & 21.0 & 21.2 \\
\hline $\mathrm{D}_{5}$ & $2-\mathrm{OH}$ & 70 & 429 & 137 & 17.50 & 17.71 \\
\hline $\mathrm{D}_{6}$ & $3-\mathrm{OH}$ & 69 & 432 & 144 & 17.66 & 17.71 \\
\hline $\mathrm{D}_{7}$ & $4-\mathrm{OH}$ & 73 & 414 & 131 & 17.64 & 17.71 \\
\hline $\mathrm{D}_{8}$ & $4-\mathrm{CH}_{3}$ & 77 & 419 & 141 & 17.86 & 17.79 \\
\hline $\mathrm{D}_{9}$ & $3-\mathrm{CH}_{3}$ & 72 & 458 & 137 & 17.77 & 17.79 \\
\hline $\mathrm{D}_{10}$ & 4-Cl & 68 & 438 & 146 & 16.85 & 17.05 \\
\hline $\mathrm{D}_{11}$ & 2,4-di $\mathrm{NO}_{2}$ & 60 & 425 & 164 & 20.05 & 20.43 \\
\hline $\mathrm{D}_{12}$ & 2,4-di $\mathrm{NO}_{2}, 6-\mathrm{Cl}$ & 62 & 484 & 178 & 19.28 & 19.22 \\
\hline $\mathrm{D}_{13}$ & 2,6-di $\mathrm{Br}, 4-\mathrm{NO}_{2}$ & 65 & 421 & 183 & 14.72 & 14.83 \\
\hline
\end{tabular}<smiles>Nc1ccc([N+](=O)[O-])cc1</smiles>

1<smiles>Nc1nc(-c2ccccc2)cs1</smiles>

Condensation<smiles>O=C(Nc1ccc([N+](=O)[O-])cc1)Nc1nc(-c2ccccc2)cs1</smiles>

2<smiles>[R]c1ccc(N)cc1</smiles>
Diazotised and coupling with 2<smiles>[R]c1ccc(N=Nc2sc(NCC(=O)Nc3ccc([N+](=O)[O-])cc3)nc2-c2ccccc2)cc1</smiles>

Here,

$\mathrm{R}=\mathrm{H},-\mathrm{OH},-\mathrm{CH}_{3},-\mathrm{NO}_{2}, \mathrm{Cl}, \mathrm{Br}$

FIgURE 1: Reaction scheme.

$(50 \mathrm{~mL})$ and washed with $5 \% \mathrm{NaHCO}_{3}$ and subsequently with water. The crude product was dried and crystallized from ethanol; it yielded pale yellow solid 2-chloro-N-(4nitrophenyl)acetamide (1). Yield: $72 \%$, M.P.: $116^{\circ} \mathrm{C}$. IR (KBr, $\mathrm{cm}^{-1}$ ): 3339 (N-H str.), 3012 (C-H str.), 1681 (C=O str.), 1514 ( $\mathrm{NO}_{2}$ str.), 741 (C-Cl); ${ }^{1} \mathrm{H}$ NMR (399.76 MHz, DMSO) $\delta$, ppm: $3.30\left(\mathrm{~s}, 2 \mathrm{H},-\mathrm{COCH}_{2}\right), 4.43(\mathrm{~s}, 1 \mathrm{H},-\mathrm{NH}), 6.81-7.65$ (m, 4H, Ar-H).

2-chloro-N-(4-nitrophenyl)acetamide (1) (4.28 gm 0.02 mole) and 2-amino-4-phenyl thiazole (3.52 gm 0.02 mole) in $20 \mathrm{~mL}$ dry benzene were refluxed for 4 hours. Benzene was removed in vacuo and crude product n-(4-nitrophenyl)-2[(4-phenyl-1,3-thiazol-2-yl)amino] acetamide (2) was dried and recrystallised from ethanol. Yield: $68 \%$, M.P.: $119^{\circ}$ C. Ca: IR (KBr, cm $\left.{ }^{-1}\right) 3328$ (N-H str.), 2993 (C-H str. aromatic ), 1685 ( $\mathrm{C}=\mathrm{O}$ str.), 1512 (C-S-C str.), 776 (C-S str. in thiazole); ${ }^{1} \mathrm{H}$ NMR (399.76 MHz, DMSO), $\delta$, ppm: 3.32 (s, $\left.2 \mathrm{H},-\mathrm{COCH}_{2}\right), 4.41$ ( $\mathrm{s}, 1 \mathrm{H},-\mathrm{NH}$ attached with thiazole ring), $6.78(\mathrm{~s}, 1 \mathrm{H},-\mathrm{NH}$ attached with aromatic ring), $6.13(\mathrm{~s}, 1 \mathrm{H}$, thiazole ring), $7.00-8.10(\mathrm{~m}, 9 \mathrm{H}, \mathrm{Ar}-\mathrm{H})$.

2.3. Diazotization and Coupling Reaction. The different aryl amines (0.01 mole) dissolved in $\mathrm{HCl}(6 \mathrm{~mL}, 50 \%)$ were cooled to $0-5^{\circ} \mathrm{C}$. A solution of sodium nitrite $(0.01$ mole, $0.69 \mathrm{gm})$ in water $(4 \mathrm{~mL})$ previously cooled to $0^{\circ} \mathrm{C}$ was added dropwise 
TABLE 2: Shade, fastness properties, K/S value, $R$ value, and \% exhaustion of the dyes.

\begin{tabular}{|c|c|c|c|c|c|c|c|c|}
\hline \multirow{2}{*}{ Dye number } & \multirow{2}{*}{ Shade on polyester } & \multicolumn{2}{|c|}{ Fastness to } & \multicolumn{2}{|c|}{ Sublimation at $180^{\circ} \mathrm{C}$} & \multirow{2}{*}{$\mathrm{K} / \mathrm{S}$ value } & \multirow{2}{*}{$R$ value } & \multirow{2}{*}{$\%$ exhaustion } \\
\hline & & Light & Washing & Staining of cotton & Staining of polyester & & & \\
\hline $\mathrm{D}_{1}$ & Yellowish brown & 4 & 5 & 4 & 4 & 5.07 & 8.29 & 70.42 \\
\hline $\mathrm{D}_{2}$ & Maroon & 5 & 5 & 5 & $4-5$ & 6.85 & 6.4 & 76.30 \\
\hline $\mathrm{D}_{3}$ & Orange & $3-4$ & 4 & $4-5$ & $4-5$ & 11.53 & 4.0 & 79.90 \\
\hline $\mathrm{D}_{4}$ & Light yellow & $4-5$ & 5 & 4 & 4 & 2.97 & 12.78 & 74.32 \\
\hline $\mathrm{D}_{5}$ & Light brown & $4-5$ & 4 & 5 & 5 & 2.17 & 16.19 & 81.75 \\
\hline $\mathrm{D}_{6}$ & Reddish brown & 5 & 5 & 5 & $4-5$ & 3.96 & 10.19 & 71.60 \\
\hline $\mathrm{D}_{7}$ & Dull yellow & 5 & 5 & 5 & 4 & 3.36 & 11.61 & 77.00 \\
\hline $\mathrm{D}_{8}$ & Reddish yellow & 4 & 5 & 4 & 4 & 8.84 & 5.10 & 71.72 \\
\hline $\mathrm{D}_{9}$ & Yellowish orange & 4 & $3-4$ & 5 & 5 & 11.38 & 4.05 & 73.00 \\
\hline $\mathrm{D}_{10}$ & Yellow & $4-5$ & 5 & $3-4$ & 3 & 8.36 & 5.36 & 80.00 \\
\hline $\mathrm{D}_{11}$ & Light yellow & 5 & 5 & 5 & 4 & 3.64 & 10.9 & 66.95 \\
\hline $\mathrm{D}_{12}$ & Reddish orange & 5 & 4 & 5 & 5 & 6.48 & 6.71 & 72.5 \\
\hline $\mathrm{D}_{13}$ & Cream & $4-5$ & 5 & $4-5$ & 4 & 5.40 & 7.86 & 70.42 \\
\hline
\end{tabular}

TABLE 3: Antimicrobial screening results of disperse dyes.

\begin{tabular}{|c|c|c|c|c|c|}
\hline \multirow{3}{*}{$\begin{array}{l}\text { Dye } \\
\text { compound }\end{array}$} & \multirow{3}{*}{$\mathrm{R}$} & \multicolumn{4}{|c|}{ Zone of inhibition $(\mathrm{mm})$} \\
\hline & & \multicolumn{3}{|c|}{ Antibacterial activity } & \multirow{2}{*}{$\begin{array}{c}\text { Antifungal activity } \\
\text { C. albicans }\end{array}$} \\
\hline & & E. coli & P. aeruginosa & S. aureus & \\
\hline $\mathrm{D}_{1}$ & $\mathrm{H}$ & 10 & 11 & 17 & 12 \\
\hline $\mathrm{D}_{2}$ & $4-\mathrm{NO}_{2}$ & 11 & 13 & 16 & 13 \\
\hline $\mathrm{D}_{3}$ & $3-\mathrm{NO}_{2}$ & 10 & 14 & 18 & 15 \\
\hline $\mathrm{D}_{4}$ & $2-\mathrm{CN}, 4-\mathrm{NO}_{2}$ & 10 & 12 & 18 & 14 \\
\hline $\mathrm{D}_{5}$ & $2-\mathrm{OH}$ & 12 & 10 & 17 & 17 \\
\hline $\mathrm{D}_{6}$ & $3-\mathrm{OH}$ & 12 & 10 & 20 & 13 \\
\hline $\mathrm{D}_{7}$ & $4-\mathrm{OH}$ & 11 & 15 & 19 & 14 \\
\hline $\mathrm{D}_{8}$ & $4-\mathrm{CH}_{3}$ & 10 & 10 & 15 & 11 \\
\hline $\mathrm{D}_{9}$ & $3-\mathrm{CH}_{3}$ & 10 & 10 & 14 & 10 \\
\hline $\mathrm{D}_{10}$ & $4-\mathrm{Cl}$ & 13 & 12 & 19 & 13 \\
\hline $\mathrm{D}_{11}$ & 2,4-di $\mathrm{NO}_{2}$ & 11 & 13 & 20 & 12 \\
\hline $\mathrm{D}_{12}$ & 2,4-di $\mathrm{NO}_{2}, 6$-Cl & 12 & 11 & 21 & 11 \\
\hline $\mathrm{D}_{13}$ & 2,6-di Br,4- $\mathrm{NO}_{2}$ & 10 & 11 & 18 & 14 \\
\hline Metronidazole & & 26 & 23 & 29 & - \\
\hline Fluconazole & & - & - & - & 19 \\
\hline
\end{tabular}

maintaining the temperature at $0-5^{\circ} \mathrm{C}$; stirring was continued for an hour, with positive test of nitrous acid on starch iodide paper. Excess of nitrous acid was destroyed by adding required amount of sulphamic acid. The resulting solution was used for coupling reaction (Figure 1).

$\mathrm{N}$-(4-nitrophenyl)-2-[(4-phenyl-1, 3-thiazol-2-yl) amino] acetamide (2) (0.01 mole) was dissolved in glacial acetic acid $(30 \mathrm{~mL})$ and cooled below $5^{\circ} \mathrm{C}$. To this well stirred solution, above mentioned diazonium chloride solution was added drop wise maintaining the $\mathrm{pH} 7.5$ to 8.0 by addition of aqueous sodium acetate $(10 \% \mathrm{w} / \mathrm{v})$. The stirring was continued for 3 hours at $0-5^{\circ} \mathrm{C}$. Then reaction mixture was poured into ice to obtain dyes $D_{1}$ to $D_{13}$, these dyes were filtered and dried at $70^{\circ} \mathrm{C}$ and were recrystallized from acetone, the properties are presented in Table 1.

\section{Results and Discussion}

All the dyes showed good performance with polyester fiber. Table 2 shows moderate to fairly good light fastness. Compounds $\mathrm{D}_{2}, \mathrm{D}_{6}, \mathrm{D}_{7}, \mathrm{D}_{11}$, and $\mathrm{D}_{12}$ showed better light fastness. The wash fastness of all the compounds was also of very good order. Introduction of terminal amino group for better dispersibility observed no notable change in the percentage exhaustion. Overall, synthesised dyes gave good dyeing on polyester fibers. All the samples showed moderate activities against $E$. coli and $P$. aeruginosa. Dyes $\mathrm{D}_{3}, \mathrm{D}_{7}, \mathrm{D}_{10}$, and $\mathrm{D}_{11}$ showed good antibacterial activity against $S$. aureus, while $\mathrm{D}_{3}$ and $\mathrm{D}_{5}$ showed good antifungal activity against $C$. albicans. Standards used were Metranidazole and Flucanazole for the comparison purpose as described in Table 3. The structures 
TABLE 4: IR and NMR data of $\mathrm{D}_{1}-\mathrm{D}_{13}$ dyes.

\begin{tabular}{|c|c|c|}
\hline $\begin{array}{l}\text { Dye } \\
\text { number }\end{array}$ & $\operatorname{IR}(\mathrm{KBr}) \mathrm{cm}^{-1}$ & ${ }^{1} \mathrm{H}$ NMR (DMSO) chemical shift in $\delta$ ppm \\
\hline $\mathrm{D}_{1}$ & $\begin{array}{l}3341(\mathrm{~N}-\mathrm{H} \text { str. }), 3011(\mathrm{C}-\mathrm{H}), 1652(\mathrm{C}=\mathrm{O}), 1578(\mathrm{~N}=\mathrm{N}), 1520 \\
\left(\mathrm{NO}_{2}\right), 1442(\mathrm{C}-\mathrm{S}-\mathrm{C}), 776(\mathrm{C}-\mathrm{S})\end{array}$ & $\begin{array}{l}3.36\left(\mathrm{~s}, 2 \mathrm{H},-\mathrm{COCH}_{2}\right), 4.30(\mathrm{~s}, 1 \mathrm{H},-\mathrm{NH}), 6.92(\mathrm{~s}, 1 \mathrm{H},-\mathrm{NH}) \\
7.37-8.48(\mathrm{~m}, 14 \mathrm{H}, \mathrm{Ar}-\mathrm{H})\end{array}$ \\
\hline $\mathrm{D}_{2}$ & $\begin{array}{l}3329(\mathrm{~N}-\mathrm{H}), 3008(\mathrm{C}-\mathrm{H}), 1660(\mathrm{C}=\mathrm{O}), 1581(\mathrm{~N}=\mathrm{N}), 1514 \\
\left(\mathrm{NO}_{2}\right), 1434(\mathrm{C}-\mathrm{S}-\mathrm{C}), 781(\mathrm{C}-\mathrm{S})\end{array}$ & $\begin{array}{l}3.34\left(\mathrm{~s}, 2 \mathrm{H},-\mathrm{COCH}_{2}\right), 4.32(\mathrm{~s}, 1 \mathrm{H},-\mathrm{NH}), 6.86(\mathrm{~s}, 1 \mathrm{H},-\mathrm{NH}) \\
7.20-8.31(\mathrm{~m}, 14 \mathrm{H}, \mathrm{Ar}-\mathrm{H})\end{array}$ \\
\hline $\mathrm{D}_{3}$ & $\begin{array}{l}3337(\mathrm{~N}-\mathrm{H}), 3007(\mathrm{C}-\mathrm{H}), 1655(\mathrm{C}=\mathrm{O}), 1578(\mathrm{~N}=\mathrm{N}), 1517 \\
\left(\mathrm{NO}_{2}\right), 1419(\mathrm{C}-\mathrm{S}-\mathrm{C}), 779(\mathrm{C}-\mathrm{S})\end{array}$ & $\begin{array}{l}3.35\left(\mathrm{~s}, 2 \mathrm{H},-\mathrm{COCH}_{2}\right), 4.35(\mathrm{~s}, 1 \mathrm{H},-\mathrm{NH}), 6.87(\mathrm{~s}, 1 \mathrm{H},-\mathrm{NH}) \\
7.15-8.21(\mathrm{~m}, 12 \mathrm{H}, \mathrm{Ar}-\mathrm{H}), 8.59(\mathrm{~s}, 1 \mathrm{H})\end{array}$ \\
\hline $\mathrm{D}_{4}$ & $\begin{array}{l}3319(\mathrm{~N}-\mathrm{H}), 3010(\mathrm{C}-\mathrm{H}), 2224(\mathrm{C} \equiv \mathrm{N}), 1664(\mathrm{C}=\mathrm{O}), 1581 \\
(\mathrm{~N}=\mathrm{N}), 1510\left(\mathrm{NO}_{2}\right), 1415(\mathrm{C}-\mathrm{S}-\mathrm{C}), 778(\mathrm{C}-\mathrm{S})\end{array}$ & $\begin{array}{l}3.32\left(\mathrm{~s}, 2 \mathrm{H},-\mathrm{COCH}_{2}\right), 4.31(\mathrm{~s}, 1 \mathrm{H},-\mathrm{NH}), 6.84(\mathrm{~s}, 1 \mathrm{H},-\mathrm{NH}) \\
7.12-8.22(\mathrm{~m}, 11 \mathrm{H}, \mathrm{Ar}-\mathrm{H}), 8.78(\mathrm{~s}, 1 \mathrm{H})\end{array}$ \\
\hline $\mathrm{D}_{5}$ & $\begin{array}{l}3322(\mathrm{~N}-\mathrm{H}), 3202(\mathrm{O}-\mathrm{H}), 2998(\mathrm{C}-\mathrm{H}), 1661(\mathrm{C}=\mathrm{O}), 1584 \\
(\mathrm{~N}=\mathrm{N}), 1511\left(\mathrm{NO}_{2}\right), 1417(\mathrm{C}-\mathrm{S}-\mathrm{C}), 1181(\mathrm{O}-\mathrm{C}), 774(\mathrm{C}-\mathrm{S})\end{array}$ & $\begin{array}{l}3.34\left(\mathrm{~s}, 2 \mathrm{H},-\mathrm{COCH}_{2}\right), 4.28(\mathrm{~s}, 1 \mathrm{H},-\mathrm{NH}), 5.64(\mathrm{~s}, 1 \mathrm{H},-\mathrm{OH}) \\
6.82(\mathrm{~s}, 1 \mathrm{H},-\mathrm{NH}), 7.10-8.27(\mathrm{~m}, 13 \mathrm{H}, \mathrm{Ar}-\mathrm{H})\end{array}$ \\
\hline $\mathrm{D}_{6}$ & $\begin{array}{l}3317(\mathrm{~N}-\mathrm{H}), 3209(\mathrm{O}-\mathrm{H}), 3007(\mathrm{C}-\mathrm{H}), 1669(\mathrm{C}=\mathrm{O}), 1580 \\
(\mathrm{~N}=\mathrm{N}), 1514\left(\mathrm{NO}_{2}\right), 1416(\mathrm{C}-\mathrm{S}-\mathrm{C}), 1176(\mathrm{O}-\mathrm{C}), 776(\mathrm{C}-\mathrm{S})\end{array}$ & $\begin{array}{l}3.31\left(\mathrm{~s}, 2 \mathrm{H},-\mathrm{COCH}_{2}\right), 4.32(\mathrm{~s}, 1 \mathrm{H},-\mathrm{NH}), 5.67(\mathrm{~s}, 1 \mathrm{H},-\mathrm{OH}) \\
6.79(\mathrm{~s}, 1 \mathrm{H},-\mathrm{NH}), 7.15-8.22(\mathrm{~m}, 13 \mathrm{H}, \mathrm{Ar}-\mathrm{H})\end{array}$ \\
\hline $\mathrm{D}_{7}$ & $\begin{array}{l}3324(\mathrm{~N}-\mathrm{H}), 3205(\mathrm{O}-\mathrm{H}), 3002(\mathrm{C}-\mathrm{H}), 1667(\mathrm{C}=\mathrm{O}), 1584 \\
(\mathrm{~N}=\mathrm{N}), 1510\left(\mathrm{NO}_{2}\right), 1414(\mathrm{C}-\mathrm{S}-\mathrm{C}), 1177(\mathrm{O}-\mathrm{C}), 768(\mathrm{C}-\mathrm{S})\end{array}$ & $\begin{array}{l}3.35\left(\mathrm{~s}, 2 \mathrm{H},-\mathrm{COCH}_{2}\right), 4.29(\mathrm{~s}, 1 \mathrm{H},-\mathrm{NH}), 5.59(\mathrm{~s}, 1 \mathrm{H},-\mathrm{OH}) \\
6.80(\mathrm{~s}, 1 \mathrm{H},-\mathrm{NH}), 7.10-8.21(\mathrm{~m}, 13 \mathrm{H}, \mathrm{Ar}-\mathrm{H})\end{array}$ \\
\hline $\mathrm{D}_{8}$ & $\begin{array}{l}3334(\mathrm{~N}-\mathrm{H}), 3208\left(\mathrm{CH}_{3}\right), 3012(\mathrm{C}-\mathrm{H}), 1669(\mathrm{C}=\mathrm{O}), 1579 \\
(\mathrm{~N}=\mathrm{N}), 1509\left(\mathrm{NO}_{2}\right), 1411(\mathrm{C}-\mathrm{S}-\mathrm{C}), 775(\mathrm{C}-\mathrm{S})\end{array}$ & $\begin{array}{l}2.59\left(\mathrm{~s}, 3 \mathrm{H},-\mathrm{CH}_{3}\right), 3.39\left(\mathrm{~s}, 2 \mathrm{H},-\mathrm{COCH}_{2}\right), 4.26(\mathrm{~s}, 1 \mathrm{H},-\mathrm{NH}) \\
6.81(\mathrm{~s}, 1 \mathrm{H},-\mathrm{NH}), 7.06-8.28(\mathrm{~m}, 13 \mathrm{H}, \mathrm{Ar}-\mathrm{H})\end{array}$ \\
\hline $\mathrm{D}_{9}$ & $\begin{array}{l}3327(\mathrm{~N}-\mathrm{H}), 3017\left(\mathrm{CH}_{3}\right), 2912(\mathrm{C}-\mathrm{H}), 1658(\mathrm{C}=\mathrm{O}), 1580 \\
(\mathrm{~N}=\mathrm{N}), 1512\left(\mathrm{NO}_{2}\right), 1418(\mathrm{C}-\mathrm{S}-\mathrm{C}), 769(\mathrm{C}-\mathrm{S})\end{array}$ & $\begin{array}{l}2.62\left(\mathrm{~s}, 3 \mathrm{H},-\mathrm{CH}_{3}\right), 3.37\left(\mathrm{~s}, 2 \mathrm{H},-\mathrm{COCH}_{2}\right), 4.31(\mathrm{~s}, 1 \mathrm{H},-\mathrm{NH}) \\
6.70(\mathrm{~s}, 1 \mathrm{H},-\mathrm{NH}), 7.11-8.18(\mathrm{~m}, 13 \mathrm{H}, \mathrm{Ar}-\mathrm{H})\end{array}$ \\
\hline $\mathrm{D}_{10}$ & $\begin{array}{l}3318(\mathrm{~N}-\mathrm{H}), 2998(\mathrm{C}-\mathrm{H}), 1665(\mathrm{C}=\mathrm{O}), 1578(\mathrm{~N}=\mathrm{N}), 1517 \\
\left(\mathrm{NO}_{2}\right), 1413(\mathrm{C}-\mathrm{S}-\mathrm{C}), 763(\mathrm{C}-\mathrm{S}), 729(\mathrm{C}-\mathrm{Cl})\end{array}$ & $\begin{array}{l}3.30\left(\mathrm{~s}, 2 \mathrm{H},-\mathrm{COCH}_{2}\right), 4.19(\mathrm{~s}, 1 \mathrm{H},-\mathrm{NH}), 6.74(\mathrm{~s}, 1 \mathrm{H},-\mathrm{NH}) \\
7.05-8.20(\mathrm{~m}, 13 \mathrm{H}, \mathrm{Ar}-\mathrm{H})\end{array}$ \\
\hline $\mathrm{D}_{11}$ & $\begin{array}{l}3305(\mathrm{~N}-\mathrm{H}), 3009(\mathrm{C}-\mathrm{H}), 1663(\mathrm{C}=\mathrm{O}), 1583(\mathrm{~N}=\mathrm{N}), 1512 \\
\left(\mathrm{NO}_{2}\right), 1416(\mathrm{C}-\mathrm{S}-\mathrm{C}), 766(\mathrm{C}-\mathrm{S})\end{array}$ & $\begin{array}{l}3.32\left(\mathrm{~s}, 2 \mathrm{H},-\mathrm{COCH}_{2}\right), 4.18(\mathrm{~s}, 1 \mathrm{H},-\mathrm{NH}), 6.67(\mathrm{~s}, 1 \mathrm{H},-\mathrm{NH}) \\
7.12-8.19(\mathrm{~m}, 11 \mathrm{H}, \mathrm{Ar}-\mathrm{H}), 8.51(\mathrm{~s}, 1 \mathrm{H},)\end{array}$ \\
\hline $\mathrm{D}_{12}$ & $\begin{array}{l}3315(\mathrm{~N}-\mathrm{H}), 3004(\mathrm{C}-\mathrm{H}), 1659(\mathrm{C}=\mathrm{O}), 1582(\mathrm{~N}=\mathrm{N}), 1516 \\
\left(\mathrm{NO}_{2}\right), 1418(\mathrm{C}-\mathrm{S}-\mathrm{C}), 772(\mathrm{C}-\mathrm{S}), 724(\mathrm{C}-\mathrm{Cl})\end{array}$ & $\begin{array}{l}3.34\left(\mathrm{~s}, 2 \mathrm{H},-\mathrm{COCH}_{2}\right), 4.23(\mathrm{~s}, 1 \mathrm{H},-\mathrm{NH}), 6.73(\mathrm{~s}, 1 \mathrm{H},-\mathrm{NH}), \\
7.10-8.12(\mathrm{~m}, 9 \mathrm{H}, \mathrm{Ar}-\mathrm{H}), 8.44\left(\mathrm{~s}, 1 \mathrm{H}, \text { between }-\mathrm{NO}_{2} \text { and } \mathrm{Cl}\right) \text {, } \\
8.79\left(\mathrm{~s}, 1 \mathrm{H} \text {, between two }-\mathrm{NO}_{2} \text { group }\right) .\end{array}$ \\
\hline $\mathrm{D}_{13}$ & $\begin{array}{l}3312(\mathrm{~N}-\mathrm{H}), 3007(\mathrm{C}-\mathrm{H}), 1661(\mathrm{C}=\mathrm{O}), 1581(\mathrm{~N}=\mathrm{N}), 1521 \\
\left(\mathrm{NO}_{2}\right), 1420(\mathrm{C}-\mathrm{S}-\mathrm{C}), 767(\mathrm{C}-\mathrm{S}), 694(\mathrm{C}-\mathrm{Br})\end{array}$ & $\begin{array}{l}3.31\left(\mathrm{~s}, 2 \mathrm{H},-\mathrm{COCH}_{2}\right), 4.17(\mathrm{~s}, 1 \mathrm{H},-\mathrm{NH}), 6.79(\mathrm{~s}, 1 \mathrm{H},-\mathrm{NH}) \\
7.00-8.05(\mathrm{~m}, 9 \mathrm{H}, \mathrm{Ar}-\mathrm{H}), 8.53(\mathrm{~s}, 2 \mathrm{H}) .\end{array}$ \\
\hline
\end{tabular}

of synthesized dyes were confirmed by spectral analysis as mentioned in Table 4.

\section{Dyeing Procedure}

The dyeing of the polyester fabric samples was carried out by HTHP dyeing method $[17,18]$.

\section{Acknowledgments}

The authors are thankful to the Principal, Navyug Science College, Surat, for providing research facilities and Atul Ltd. for chemicals and dyeing facilities.

\section{References}

[1] L. Racane, R. Stojkovic, V. Tralic-Kulenovic, and G. KarminskiZamola, "Synthesis and antitumor evaluation of novel derivatives of 6-amino-2-phenylbenzothiazoles," Molecules, vol. 11, no. 5, pp. 325-333, 2006.

[2] B. R. Modi, D. M. Vashi, and K. R. Desai, "Synthesis of 8-triazinylamino coumarin derivatives and their fluorescent properties," Indian Journal of Chemical Technology, vol. 1, no. 5, pp. 317-318, 1994.

[3] I. Sigmundová, P. Zahradník, P. Magdolen, and H. Bujdáková, "Synthesis and study of new antimicrobial benzothiazoles substituted on heterocyclic ring," Arkivoc, vol. 2008, no. 8, pp. 183-192, 2008.

[4] D. W. Rangnekar and J. B. Mehta, "Synthesis and dyeing performance of 1-arylazo-3-(-4-substituted benzamido)naphth-2-ols and 1-arylazonaphth-2-ol-3, 6-disulphonamide," Indian Journal of Fiber and Textile Research, vol. 18, pp. 145-150, 1993.

[5] A.D. Towns, Developments in azo disperse dyes derived from heterocyclic diazo components, vol. 42, no. 1, pp. 3-28, 1999.

[6] D. W. Rangnekar, V. R. Kanetkar, G. S. Shankarling, and J. V. Malanker, "Synthesis and application of 2-arylazo-5-[styryl4-(B-cyano/carbethoxy-b-cyanoethenyl)]-1,3,4-thiadiazoles," Journal of Heterocyclic Chemistry, vol. 36, p. 95, 1999.

[7] H. R. Maradiya, "Monoazo disperse dyes based on 2-amino1,3,4-thiadiazole derivatives," Journal of The Serbian Chemical Society, vol. 67, no. 11, pp. 709-718, 2002.

[8] P. C. Miranda, L. M. Rodrigues, M. S. T. Goncalves, S. P. G. Costa, R. Hardina, and A. M. F. Oliveira, "Synthesis, wash and light fastness of azo dyes derived from N,N-diethylanilines," Advance in Colour Sceince and Technology, vol. 4, no. 1, 2001.

[9] M. A. Metwally, E. Abdel-Latif, F. A. Amer, and G. Kaupp, "Synthesis of new 5-thiazolyl azo-disperse dyes for dyeing polyester fabrics," Dyes and Pigments, vol. 60, no. 3, pp. 249-264, 2004.

[10] H.-L. Liu, Z. Li, and T. Anthonsen, "Synthesis and fungicidal activity of 2-Imino-3-(4-arylthiazol-2-yl)-thiazolidin-4ones and their 5-arylidene derivatives," Molecules, vol. 5, no. 9, pp. 1055-1061, 2000. 
[11] A. A. Chavan and N. R. Pai, "Synthesis and Biological Activity of N-Substituted-3-chloro-2-azetidinones," Molecules, vol. 12, no. 11, pp. 2467-2477, 2007.

[12] S. K. Sonwane, S. D. Srivastava, and S. K. Srivastava, "Synthesis and antimicrobial activity of 2-( 2 ' -arylidene-hydrazino-acetylamino)-4-phenyl-1,3-thiazoles and 2-[2'-4" -substituted-aryl$3^{\prime \prime}$-chloro-2" -oxo-azetidine-acetyl-amino]-4-phenyl-1,3thiazoles," Indian Journal of Chemistry B, vol. 47, no. 4, pp. 633-636, 2008.

[13] S. M. Mohamed, "Synthesis of fused coumarino-[4,3-d]-pyrimidine derivatives," Indian Journal of Heterocyclic Chemistry, pp. 67-68, 2005.

[14] M. J. Ladani, S. D. Tala, J. D. Akbari, M. F. Dhaduk, and H. S. Joshi, "Synthesis and biological study of oxopyrimidines and thiopyrimidines of 2-(2,4-dichlorophenyl)imidazo[1,2a]pyridin-3-carbaldehyde," Journal of the Indian Chemical Society, vol. 86, no. 1, pp. 104-108, 2009.

[15] K. F. Ansari and C. Lal, "Synthesis and biological activity of some heterocyclic compounds containing benzimidazole and beta-lactam moiety," Journal of Chemical Sciences, vol. 121, no. 6, pp. 1017-1025, 2009.

[16] D. P. Bhoot, R. C. Khunt, V. K. Shankhavara, and H. H. Parekh, "Synthesis of some new heterocyclic compounds with potential biological activity," Journal of Sciences Islamic Republic of Iran, vol. 17, no. 4, pp. 323-325, 2006.

[17] M. M. Dalal and K. R. Desai, "Synthesis of monoazo disperse dyes from 2-amino, 4-methoxy benzothiazole and their application on polyester fibers," Oriental Journal of Chemistry, vol. 11, p. 71, 1995.

[18] H. R. Maradiya and V. S. Patel, "Thiazole based disperse dyes for nylon and polyester fibers," Fibers and Polymers, vol. 2, no. 3, pp. 153-158, 2001. 

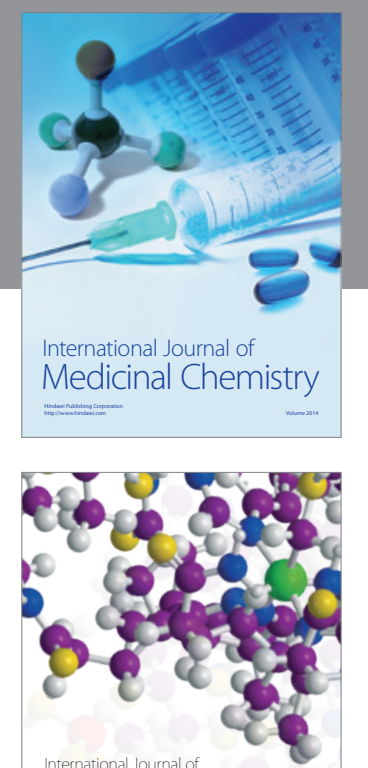

\section{Carbohydrate} Chemistry

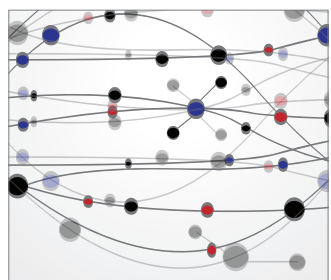

The Scientific World Journal
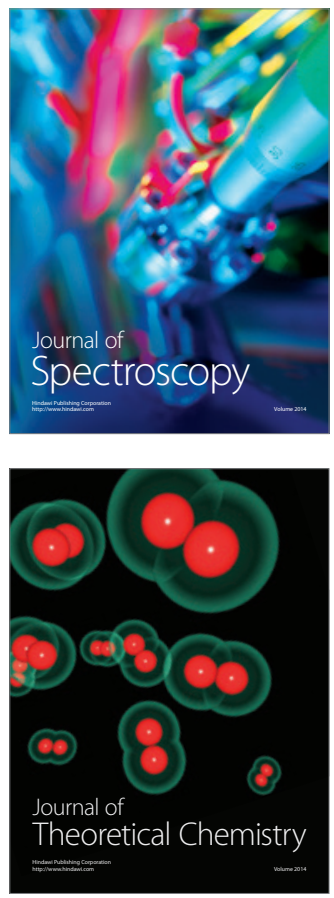
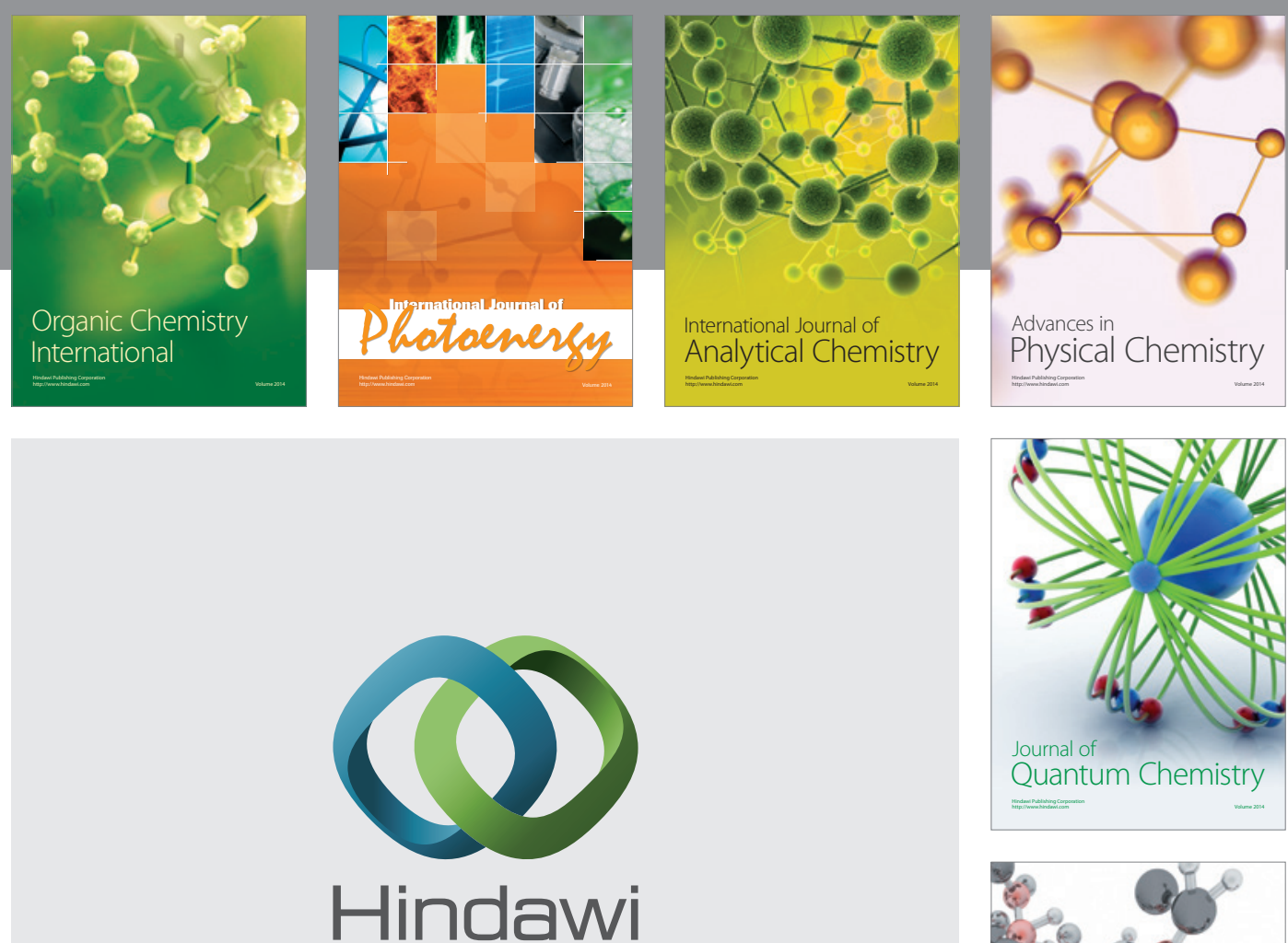

Submit your manuscripts at

http://www.hindawi.com

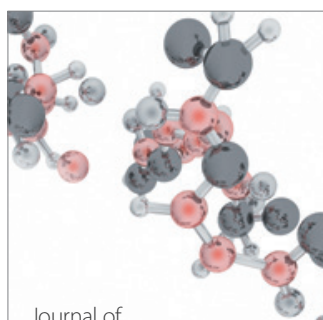

Analytical Methods

in Chemistry

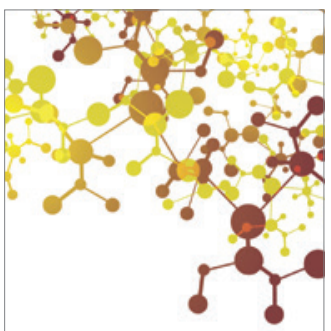

Journal of

Applied Chemistry

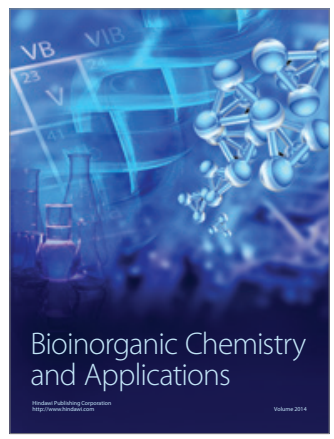

Inorganic Chemistry
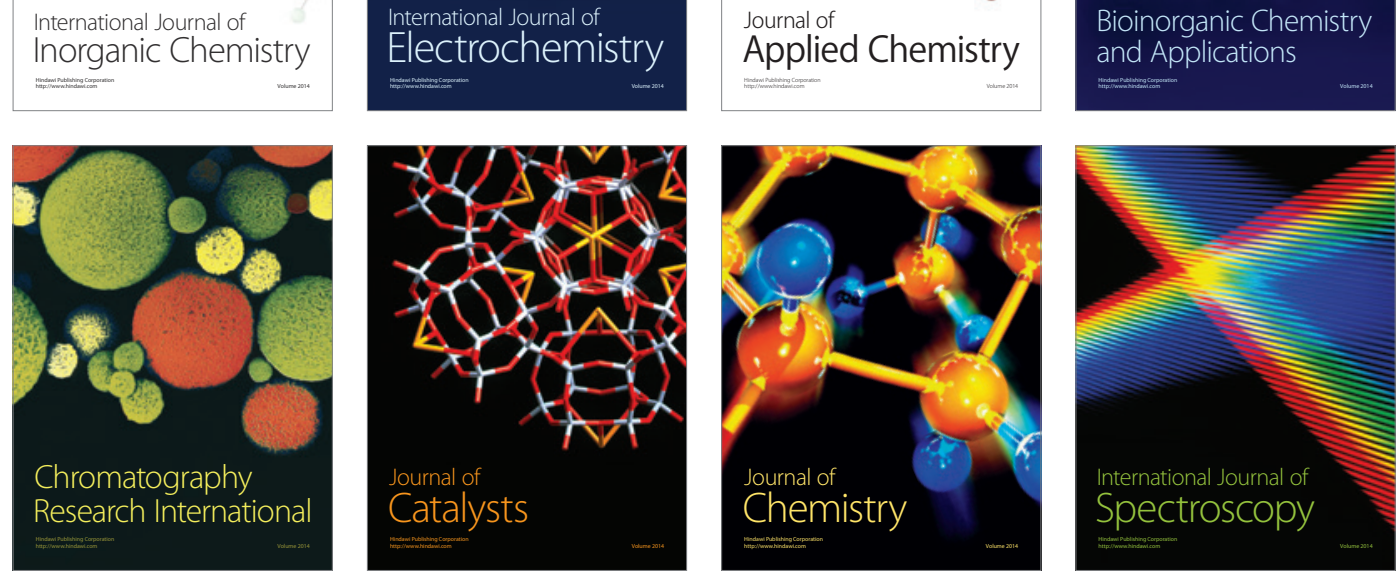